\title{
Effect of phosphorus concentration on the atomic distribution function of amorphous Pd-Ni-P alloys*
}

\author{
Jean Dixmier ${ }^{\dagger}$ and Pol Duwez \\ W. M. Keck Laboratory of Engineering Materials, California Institute of Technology, Pasadena, \\ California 91109. \\ (Received 25 August 1972) \\ The effect of the phosphorus concentration on the atomic distribution function in amorphous alloys \\ $\left(\mathrm{Pd}_{50}-\mathrm{Ni}_{50}\right)_{100-x} \mathbf{P}_{x}$ in which $x$ varies from 15 to 27.5 was investigated. The alloys were obtained by \\ rapid quenching from the liquid state. The intensity of the first peak of the interference function \\ decreases with increasing phosphorus content. The second peak has a definite shoulder on the right \\ side for alloys low in phosphorus and this shoulder progressively disappears as the $\mathrm{P}$ concentration \\ is increased. This feature was not found before in binary Ni-P alloys obtained by electrodeposition. \\ From the atomic distribution functions it was possible to determine the metal-to-metal distances and \\ the approximate metal-to-phosphorus distances.
}

\section{INTRODUCTION}

Many amorphous alloys obtained by rapid quenching from the liquid state have been reported. The structure of these alloys has been investigated mostly by $\mathrm{x}$-ray diffraction techniques and this subject has been the object of a recent review. ${ }^{1}$ It is generally accepted that these alloys are amorphous (glassy) and not microcrys talline. A glass transition temperature has been measured by Chen and Turnbull. ${ }^{2} \mathrm{~A}$ discussion on how to differentiate between these two classes of metallic solids has been published by Dixmier and Guinier. ${ }^{3}$ As pointed out by Cargill, ${ }^{4}$ the $\mathrm{x}$-ray diffraction patterns and the interference functions of all amorphous metallic alloys look very similar and this may lead to the tentative conclusion that the short-range order might be the same in all alloys. A quasicrystalline model based on the crystal structure of the phosphides $\mathrm{Ni}_{3} \mathrm{P}$ and $\mathrm{Fe}_{3} \mathrm{P}$ was used by Maitrepierre, ${ }^{5}$ and this model did not lead to a very good agreement between the experimental results and the calculated atomic distribution functions. A weakness of this approach is that it assumes that all the atoms (the metal as well as the semimetal) are displaced independently of each others. This hypothesis is probably not correct because the nonmetallic atom (generally $\mathrm{Si}$, $P$, or $B$ ) whose concentration is always around $20 \pm 5$ at. \% must play an important role in promoting the amorphous nature of the alloys. In binary alloys, the concentration range of the semimetals is generally restricted, except in the case of NiP alloys obtained by electrolysis. ${ }^{4}$ In ternary alloys containing phosphorus, the widest range for phosphorus (15 and 25.5 at. \%) has been found in Pd-Ni-P alloys ${ }^{5}$ and the effect of the $\mathbf{P}$ concentration on the electrical resistivity has been studied by Boucher. ${ }^{6}$ The present study is concerned with the effect of $P$ concentration on the structure of $\mathrm{Pd}-\mathrm{Ni}-\mathrm{P}$ alloys containing equiatomic concentrations of $\mathrm{Pd}$ and Ni.

\section{EXPERIMENTAL PROCEDURES}

The experimental procedures were essentially the same as those described in Ref. 5, 7, and 8. Powders of the three constituants are mixed, pressed in a $\frac{1}{4}-i$. steel die at $50000 \mathrm{psi}$. The compacts are sealed in evacuated Pyrex capsules and heated up to $500^{\circ} \mathrm{C}$ at an average rate of $100^{\circ} \mathrm{C}$ per day, which is slow enough to allow the phosphorus to react with the metal powders and form stable phosphides by solid-state reaction. The compacts are then sealed in quartz capsules and heated to a temperature near the melting point (about $750{ }^{\circ} \mathrm{C}$ ) for about two days. The alloys are then melted by induction under an argon atmosphere and sucked into $2-\mathrm{mm}$ quartz tubings. About $100 \mathrm{mg}$ of the alloys are then rapidly quenched from the liquid state by the piston and anvil method. ${ }^{9}$ The quenched foils are about $40 \mu \mathrm{m}$ thick and 20-25 $\mathrm{mm}$ in diameter. Their amorphous structure is carefully checked by scanning the first diffraction maximum with $\mathrm{Cu} K_{\alpha}$ radiation between $2 \theta$ angles of about $30^{\circ}$ and $50^{\circ}$. The diffractometer is moved at a rate of

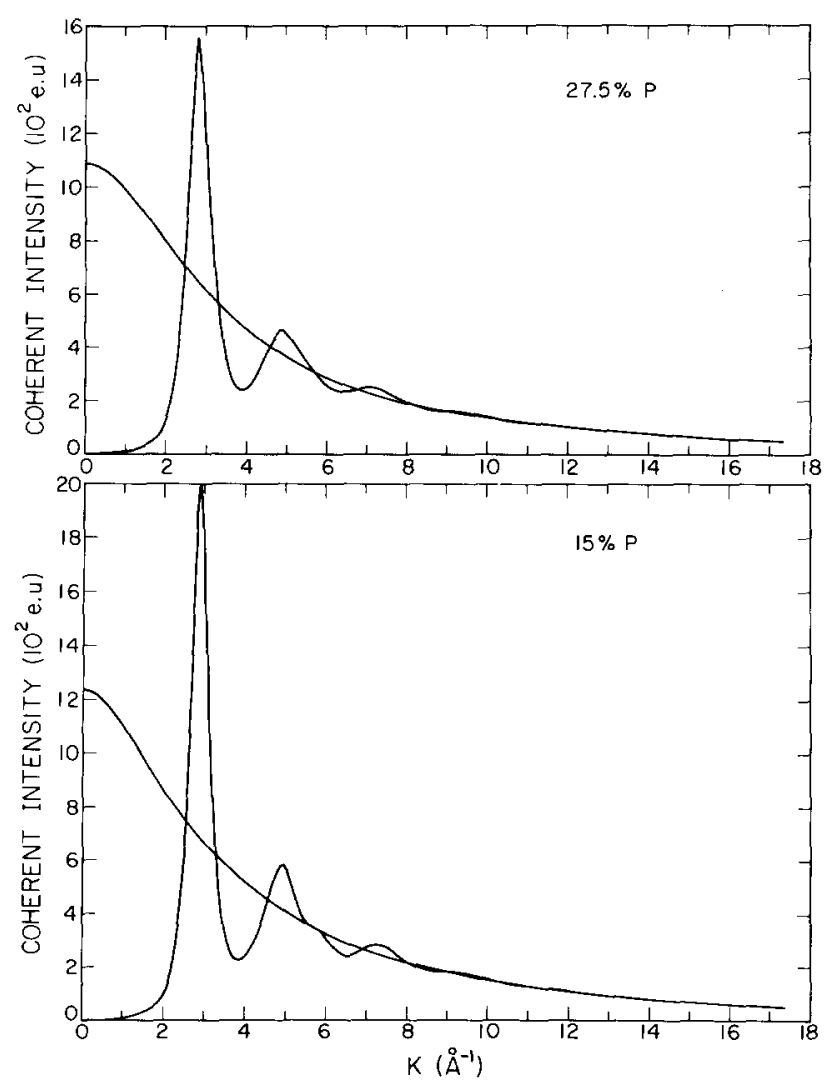

FIG. 1. Typical coherent intensity vs $K$ curves for two alloys containing the largest and the smallest concentration of phosphorus. 


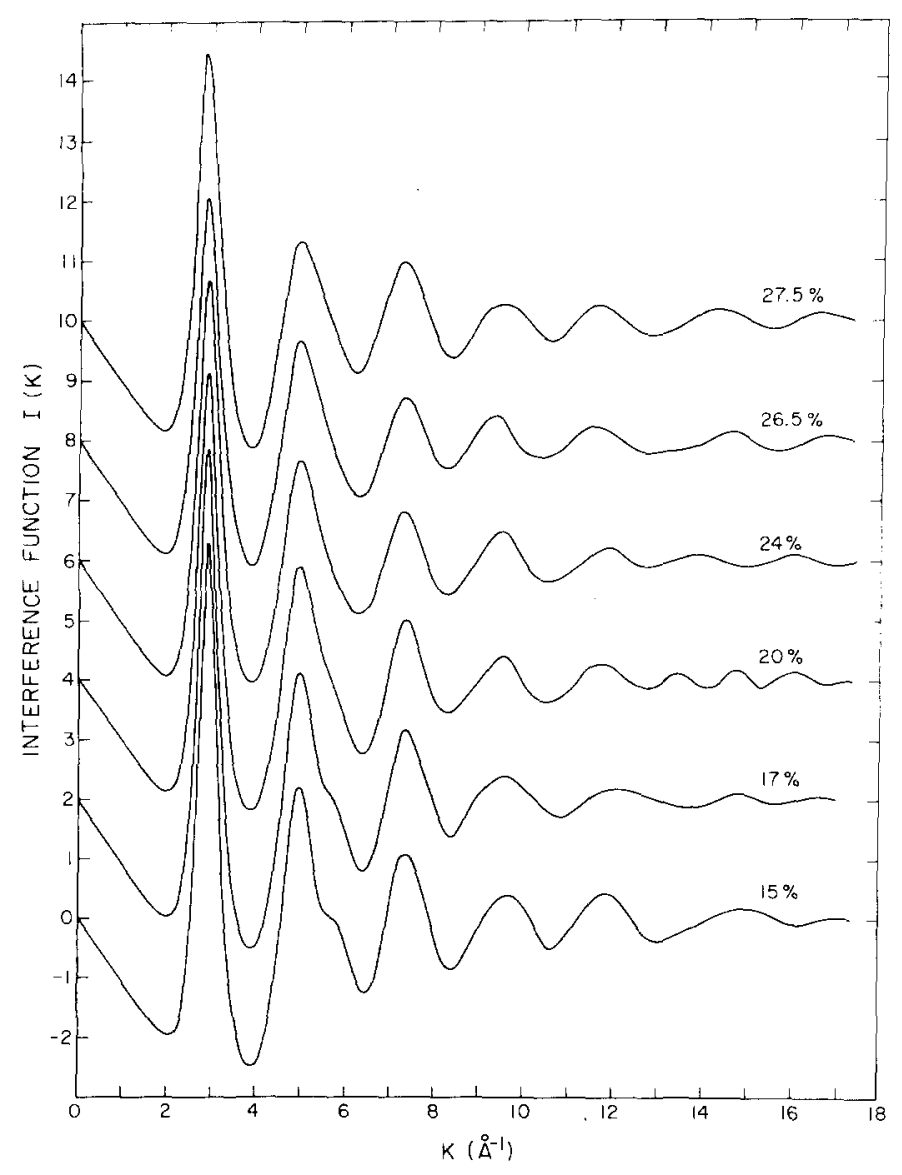

FIG. 2. Interference function for alloys with various phosphorus concentrations.

$0.04^{\circ} / \mathrm{min}$ and an intensity reading is automatically plotted on a chart recorder every $100 \mathrm{sec}$, which corresponds to a $2 \theta$ interval of $0.066^{\circ}$. This scan is accurate enough to detect the presence of small crystalline regions and the foil is rejected if this happens.

The complete $\mathrm{x}$-ray diffraction patterns between $2 \theta$ angles from $12^{\circ}$ to $160^{\circ}$ were obtained with a G.E. diffractometer, using Mo $K_{\alpha}$ radiation with a LiF curved crystal monochromator located on the diffracted beam. The specimens consisted of three foils glued together with a small layer of Duco cement on a bakelite substrate. Such specimens may be considered as being infinitely thick for the purpose of absorption corrections. Between $2 \theta$ angles of $12^{\circ}-70^{\circ}$ the diffractometer moved at a rate of $0.02^{\circ}$ in $100 \mathrm{sec}$, and an intensity reading was printed on a paper tape every $1000 \sec \left(2 \theta=0.2^{\circ}\right)$. From $70^{\circ}$ to $160^{\circ}$ in $2 \theta$, the rate of scanning was doubled so that a reading was obtained every $0.4^{\circ}$ in $2 \theta$. The total time for a complete scan is about $138 \mathrm{~h}$. Before the data are fed into a computer, the intensity-vs $-2 \theta$ curves are plotted on a large-scale graph paper to make sure that the curve is very smooth and no failure occurred in the electronic recording equipment.

\section{ANALYSIS OF DATA}

The intensity -vs-2 $\theta$ curves were corrected for polarization and background by the usual methods. Since seven intensity maxima were detected in these curves, special care was taken in the normalization procedure to obtain the intensities in electron units. The normalization constant is generally determined by the high angle method ${ }^{10}$ in which the average value of the function $i(K)$, in which $K=(4 \pi \sin \theta) / \lambda$, is matched with the function $\Sigma_{m} x_{m} f_{m}^{2}$, in which $x$ is the atomic concentration of the $m$ atoms and $f_{m}$ is their scattering factor. In most studies, the normalization constant is determined at a $K$ value around $10 \AA^{-1}$. In the present case, the measured intensities at high angle were reliable enough to normalize up to a $K$ value of about $17.5 \AA^{-1}$. This was done by tracing a smooth curve so that the observed $i(K)$ curve with the peaks Nos. $4-7$ would oscillate around the theoretical curve. The normalization constant obtained at the point at which the two curves were crossing was first taken for the part of the curve below a $K$ value of about 9 , and then was changed by about 1 or $2 \%$ to obtain the best fit with the experimental curve. A computer program for the IBM $370 / 155$ was used for determining the interfer ence function

$$
I(K)=K[a(K)-1]
$$

and the atomic distribution function

$$
W(r)=2 / \pi \int_{0}^{\infty} I(K) \sin K r d K
$$
with

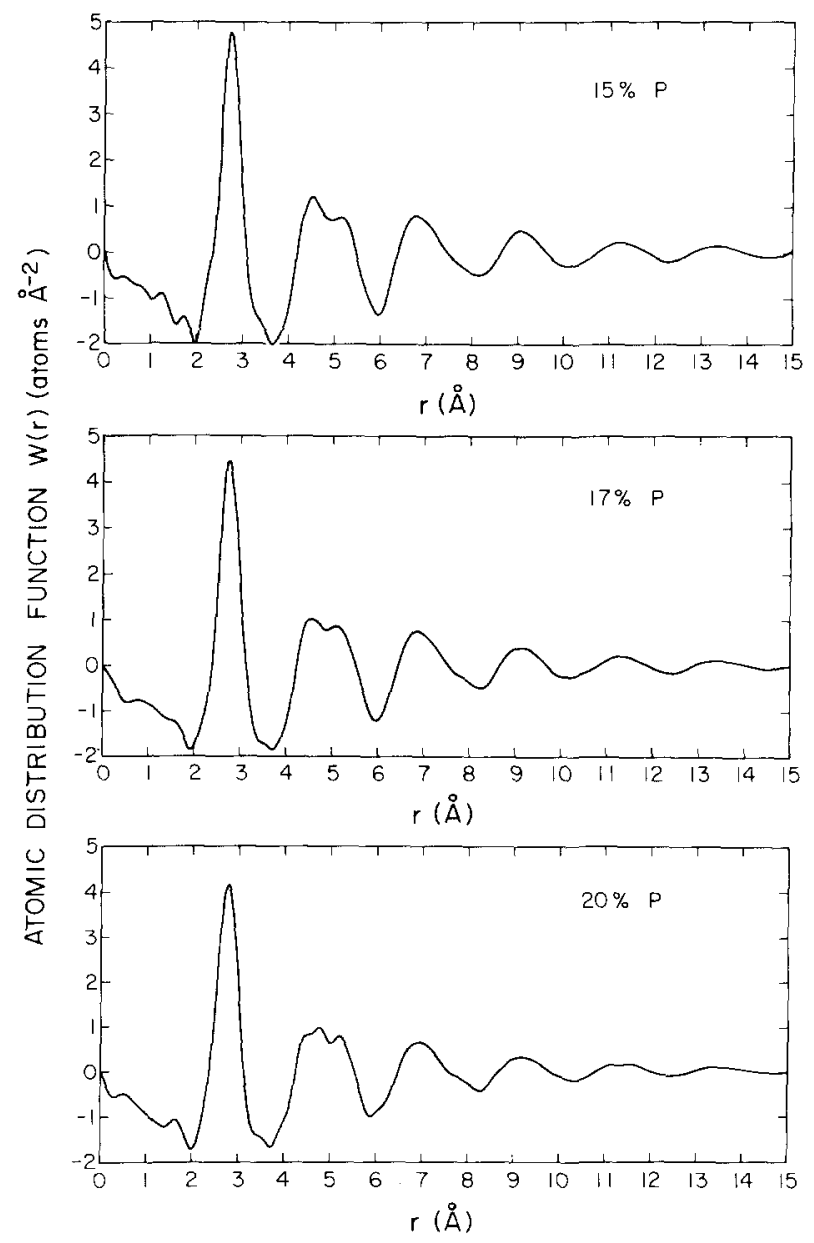

FIG. 3. Atomic distribution functions for alloys containing 15 , 17 , and 20 at. $\%$ phosphorus. 

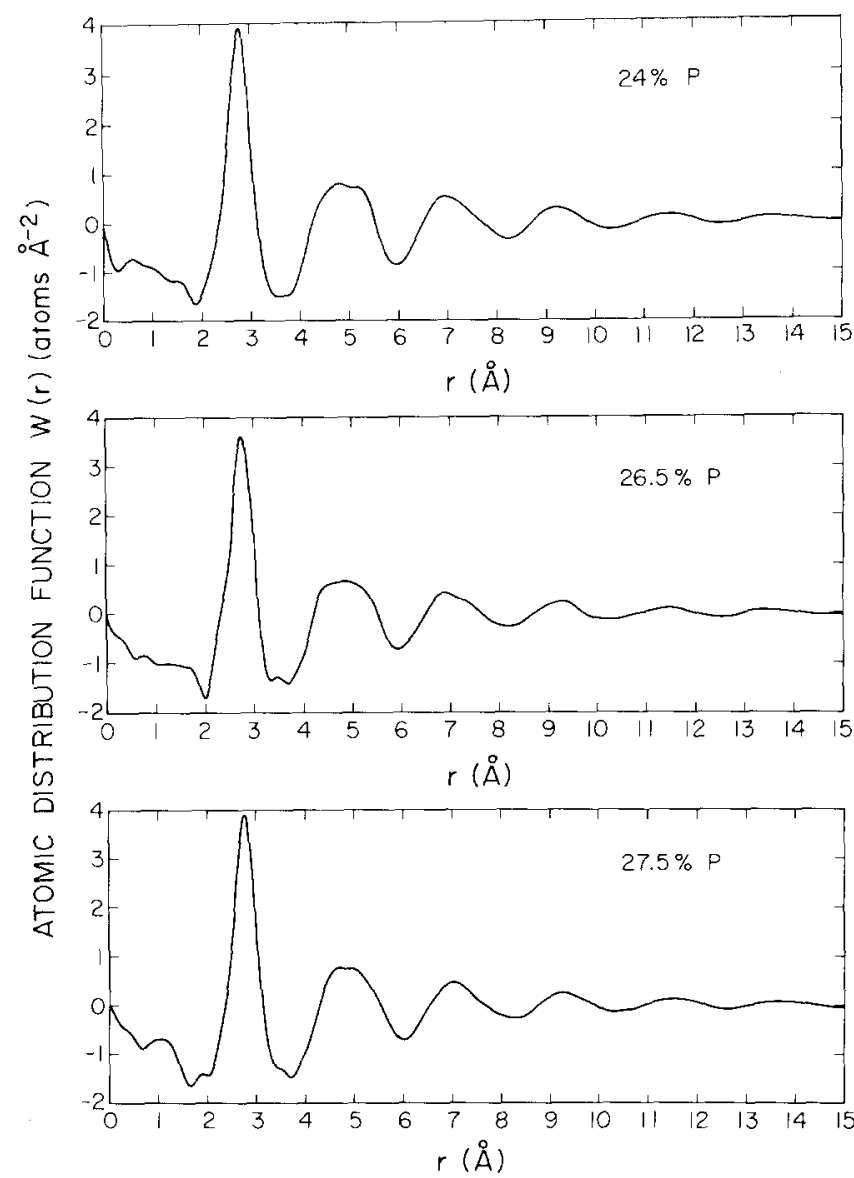

FIG. 4. Atomic distribution functions for alloys containing 24 , 26.5 , and 27.5 at. $\%$ phosphorus.

$$
a(K)=1+\left(I_{c}^{\text {eu }}-\Sigma_{m} x_{m} f_{m}^{2}\right) /\left(\Sigma_{m} x_{m} f_{m}\right)^{2},
$$

where $I_{c}^{\mathrm{eu}}$ is the normalized coherent intensity per atom in electron units. In order to retain the information contained in the weak but real maxima measured at high angles, the damping factor used to minimize the termination errors was as small as possible. It was found that the termination ripples were completely removed with a damping factor equal to 0.002 .

\section{RESULTS}

The coherent intensity curves for the two alloys containing the smallest and largest concentrations of phosphorus are shown in Fig. 1. The maxima following the third one are not visible because of the small size of the figure. The effect of increasing phosphorus, however, is illustrated by a decrease in the intensity of the first peak, a broadening of the first and second peaks, and the disappearance of the shoulder on the right side of the second peak. The interference functions $I(K)$ are shown in Fig. 2 for the six alloys containing various amounts of phosphorus. The wiggles at high values of $K$ are due to uncertainties in the normalization procedure. It is significant to note that the shoulder on the right side of the second peak disappears between 20 and 24 at. \% P and the intensity of the first peak decreases continuously with increasing phosphorus concentration. A similar decrease in intensity has been observed in binary $\mathrm{Ni}-\mathrm{P}$ amorphous alloys obtained by electrolysis, ${ }^{4}$ but in that case the shoulder on the second peak is always present up to 26 at. $\%$ P. By using a very small damping factor which is directly related to the shortrange order, the information contained in the maxima at high angles was retained and interesting details are found in the atomic distribution functions. As shown in Figs. 3 and 4 the first peak of this function is not symmetrical and has a shoulder on the left side. To analyze this shoulder, a symmetrical curve was traced by taking the mirror image of the right side with respect to the location of the maximum (dashed lines in Figs. 5 and 6) and the difference between this curve and the observed one was plotted and is shown by the dotted lines. It is believed that the lack of symmetry in the first-neighbors peaks is due to the metal-to-nonmetal distances. Although the scattering factor of the metal atoms is

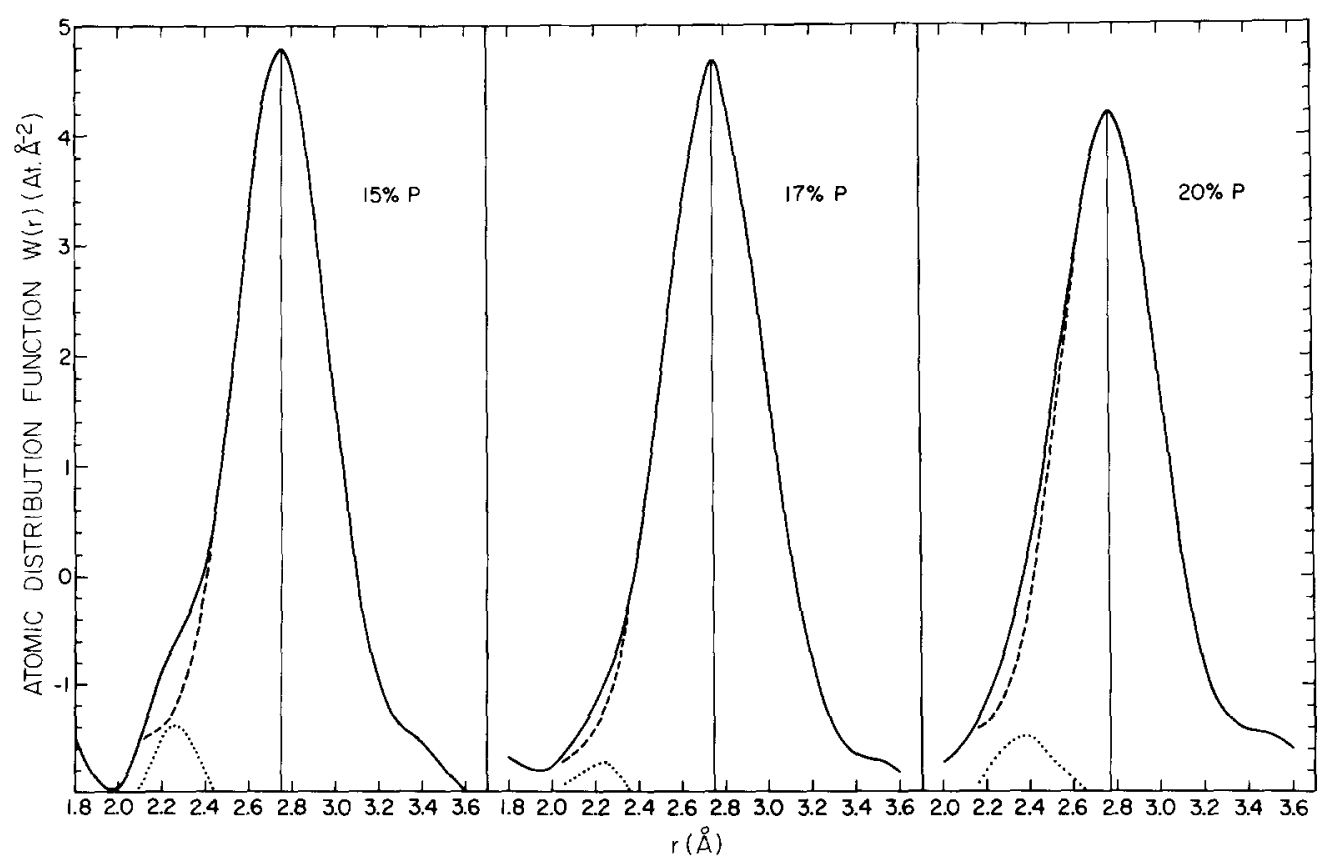

FIG. 5. First peak in the atomic distribution function for alloys containing 15.7 , and 20 at. \% phosphorus. 


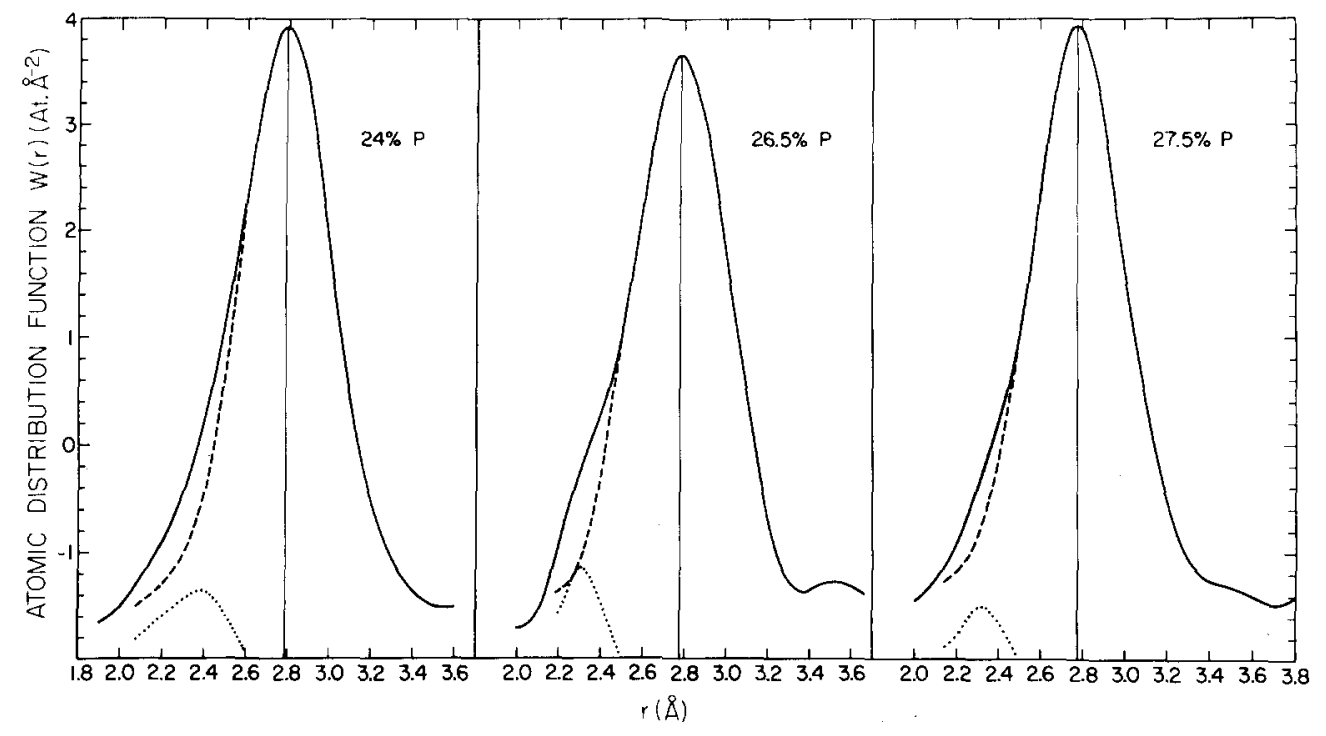

FIG, 6. First peak in the atomic distribution function for alloys containing 24 , 26.5 , and 27.5 at. $\%$ phosphorus.

greater than that of phosphorus, the contribution of the metal and nonmetal pairs to the first peak is about $10 \%$ and can indeed be observed. It might be argued that the asymmetry of the first peak is due to ripples resulting from a poor normalization in the atomic distribution function below a value of about $2 \AA$. However, even when only weak ripples exist before the first peak (alloys containing 17 and 20 at.\% P in Fig. 3), the lack of symmetry is still quite apparent in Fig. 5.

The metal-metal nearest-neighbor distances and the metal-phosphorus distances are shown in Fig. 7 as a function of $\mathbf{P}$ concentration. These two distances increase with the $\mathrm{P}$ concentration up to 22 and 23 at. $\% \mathrm{P}$ and then decrease. This seems to indicate that the presence of $\mathbf{P}$ which is smaller than the metallic atoms increases the local disorder in the alloys. The decrease above 25 at. \% P may be due to a stronger bonding between the metal atom and phosphorus similar to that in the crystalline phosphides $\mathrm{Pd}_{3} \mathrm{P}$ and $\mathrm{Ni}_{3} \mathrm{P}$.

The second peak in the atomic distribution functions shows interesting features. As shown in Fig. 3 the usual split into two maxima is present in the alloys containing 15 and 17 at.\% P. At 20 at.\% P, a third peak appears between the two maxima. This may be due to the presence of two kinds of metallic elements of different sizes with different short-range orders with phosphorus. From 24 to 27.5 at. $\%$ P, the split in the second peak gradually disappears (Fig. 4).

\section{CONCLUSIONS}

The important results of the present investigation are as follows: (i) The intensity of the first peak in the interference function decreases with increasing phosphorus content and its width (whose inverse can be regarded as a measure of the apparent grain size) increases in the same range of $P$ concentration (Fig. 2). The shoulder following the second peak in the interference function is quite pronounced at low $\mathrm{P}$ contents and progressively disappears as the $P$ concentration is increased. If, as it has been suggested, the high intensity of the first peak and the shoulder on the second peak were due to the presence of a near-covalent bonding between the metal and the semimetal, the two observed features in the interference function described above should vary in exactly the opposite direction. It is therefore difficult to explain the present results by the covalent effect of the metalloid atoms. Another possibility is to consider a random dense packing of spheres of different diameters, following Bernal's random packing of rigid spheres. Some attempts have been made in this direction by Finney ${ }^{11}$ and more recently by Bennett. ${ }^{12}$ The results of their computer calculations are given in terms of the atomic distribution function and show a well-defined split of the second peak. This splitting, however, does not mean that the interference function would show a shoulder as observed in this investigation. The interference function would provide a better test of the theory than the atomic distribution function, because it can be directly compared with experimental results.

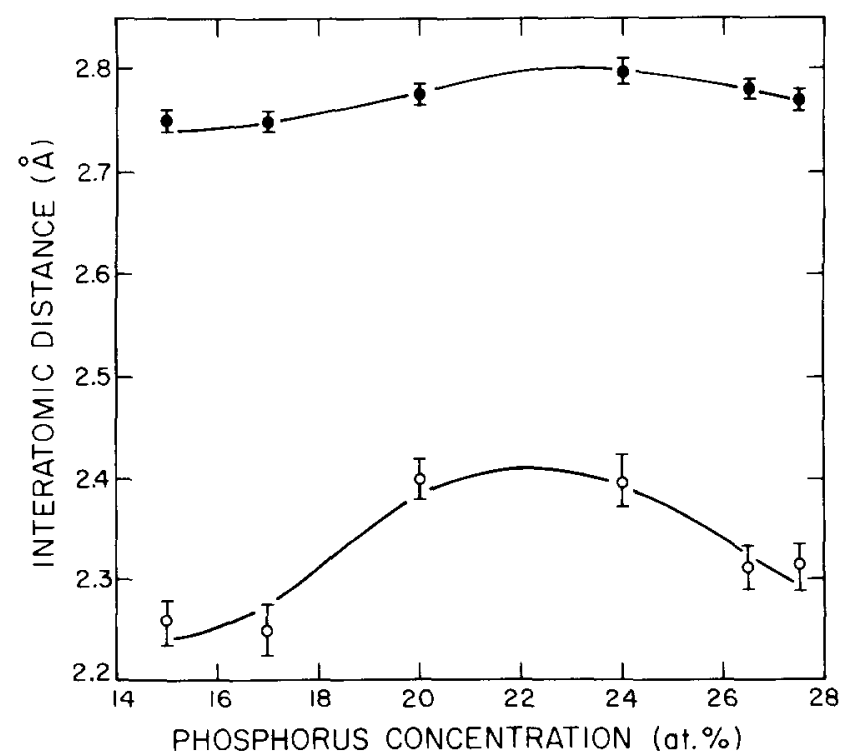

FIG. 7. Metal-to-metal (๑) and metal-to-phosphorus interatomic distances (o) vs phosphorus concentration. 
A promising approach to the problem may be a theoretical study of the interference function of random packing of hard spheres of two different diameters. This study should include the effect of the concentration of the two spheres of given diameters and the effect of the diameter ratio for a given concentration. Sadoc ${ }^{13}$ has undertaken such a study and preliminary results indicate that for $15 \%$ of a smaller sphere and a difference of $10 \%$ in size, there is a definite shoulder on the second peak of the interference function. This approach looks promising and results will be the subject of subsequent publications.

*Work supported by the U. S. Atomic Energy Commission.

'Present address: Université Paris-Sud, Laboratoire de Physique des
Solides, 91 Orsay, France.

'B. C. Giessen and C. N. J. Wagner, in Physics and Chemistry of Liquid Metals, edited by S. Beez (Marcel Dekker, New York, 1972), pp. 633-695.

${ }^{2}$ H. S. Chen and D. Turnbull, J. Appl. Phys. 38, 3646 (1967).

${ }^{3}$ J. Dixmier and A. Guinier, Fizika 2, 9 (1970).

${ }^{4}$ G. S. Cargill III, J. Appl. Phys. 41, 12 (1970).

${ }^{5}$ P. L. Maitrepierre, J. Appl. Phys. 40, 4826 (1969).

${ }^{6}$ B. Y. Boucher, J. Non-Cryst. Solids 7, 277 (1971).

${ }^{7}$ S. C. H. Lin and P. Duwez, Phys. Status Solidi 34, 469 (1969).

${ }^{8}$ A. K. Sinha and P. Duwez, J. Phys. Chem. Solids 32, 267 (1971).

${ }^{9}$ Pol Duwez, in Progress in Solid State Chemistry, edited by H. Reiss (Pergamon, London, 1966), p. 377.

${ }^{10} \mathrm{~K}$. Furukawa, Rep. Prog. Phys. 25, 395 (1962).

${ }^{11}$ J, L, Finney, Proc. R. Soc. Lond. 319, 479 (1970).

${ }^{12}$ C. H. Bennett, J. Appl. Phys. 43, 2727 (1972).

${ }^{13} \mathrm{~J}$. F. Sadoc (private communication). 